\title{
Влияние квантового магнитного поля на разогрев носителей заряда в чистом Ge
}

\author{
(C) В.Ф. Банная ${ }^{1}$, Е.В. Никитина ${ }^{2, \text { ฯ }}$ \\ ${ }^{1}$ Московский педагогический государственный университет, \\ 119991 Москва, Россия \\ ${ }^{2}$ Российский университет дружбы народов, \\ 117198 Москва, Россия \\ ฯ E-mail: enikitina@sci.pfu.edu.ru
}

(Получена 14 июня 2018 г. Принята к печати 21 февраля 2018 г.)

Подробно рассмотрены результаты экспериментального исследования разогрева носителей заряда электрическим полем $(\mathbf{E})$ в чистом германии $n$ - и $p$-типов, в квантующем магнитном поле $(\mathbf{H})$ при $\mathbf{E} \perp \mathbf{H}$, в условиях фотовозбуждения носителей. Полученные результаты качественно согласуются с теорией захвата носителей заряда в скрещенных электрическом и магнитном полях.

DOI: 10.21883/FTP.2019.01.46980.8837

\section{1. Введение}

В работах $[1,2]$ изучалось влияние магнитного поля на низкотемпературный примесный пробой в чистом Ge. Как показали оценки, в условиях электрического пробоя средняя энергия носителей такова, что даже в магнитных полях $H \approx 5 \cdot 10^{4}$ Э квантование энергии снимается. Однако в электрических полях $E<E_{\mathrm{br}}$ этот эффект может оказать существенное влияние как на механизм рассеяния энергии, так и на процессы рекомбинации. Поэтому представляет интерес изучение зависимостей удельной электропроводности и концентрации свободных носителей заряда (постоянной Холла) от величины $E$ в условиях греющего электрического и квантового магнитного полей.

\section{2. Условия и результаты эксперимента}

Условие квантования $\hbar \omega_{c}>k_{0} T\left(\omega_{c}-\right.$ циклотронная частота) при $T=4.2 \mathrm{~K}$ выполняется в $n$-Ge начиная с $H \gtrsim 5 \kappa Э$, в $p$-Ge для тяжелых дырок с $H \gtrsim 9$ кЭ. Исследовались образцы $n$ - и $p$-Ge c концентрацией ионизованных центров $N_{I} \lesssim 10^{13} \mathrm{~cm}^{-3}$, что позволяет считать превалирующим механизмом рассеяния энергии и импульса носителей на акустических колебаниях решетки. Параметры образцов приведены в таблице. (В настоящей работе используются результаты измерений, выполненные в работах [1-5]).

Измерения выполнялись при $T=4.2 \mathrm{~K}$. Свободные носители в зоне создавались фотогенерацией - фоновым подсветом (при $T \approx 300 \mathrm{~K}$ ). Интенсивность фотогенерации оставалась примерно постоянной, а ее величина $I$ позволяла пренебречь концентрацией носителей, создаваемых термовозбуждением.

Измеряемыми величинами являлись постоянная Холла $R_{\mathrm{H}}$ и удельная электропроводность $\sigma$. Величина электрического поля $E$ варьировалась от $\sim 5 \cdot 10^{-2}$ до $\sim 10^{2} \mathrm{~B} / \mathrm{cM}$, магнитного $H-$ от 0 до $\sim 5 \cdot 10^{4}$ Э.
В условиях эксперимента постоянная Холла обусловлена одним типом носителей, однако при фотовозбуждении зависимости $R_{\mathrm{H}}(E, H) \sim\left\langle\tau_{c}\right\rangle(E, H)$ обусловлены временем жизни свободных носителей. Это видно из следующих простых соображений.

В случае изолированных кулоновских центров захвата уравнение баланса частиц имеет вид

$$
I-A_{T}(E, H)\left(N_{d}-N_{a}-n\right)=0,
$$

где $I$ - полный рекомбинационный поток в единице объема; $A_{T}(E, H)$ - коэффициент термической ионизации. Величина $I$ с $n$ и $\left\langle\tau_{c}\right\rangle(E, H)$ связана соотношением

$$
I=\frac{n}{\left\langle\tau_{c}\right\rangle(E, H)} .
$$

Подставляя (2) в (1) и полагая, что $n \ll N_{d}, N_{a},\left|N_{d}-N_{a}\right|$, получим

$$
n=\frac{\left\langle\tau_{c}\right\rangle(E, H) A_{T}(E, H)}{\left|\left(N_{d}-N_{a}\right)\right|} .
$$

Из (3) видно, что при фотовозбуждении, т.е. $I \gg \frac{A_{T}(E, H)}{N_{d}-N_{a}}$, зависимость $n(E, H)$ характеризует поведение времени жизни носителей заряда (при учете термогенерации характер зависимостей $n(E, H)$ определяется еще и зависимостью $\left.A_{T}(E, H)\right)$.

Зависимости $R_{\mathrm{H}}(E, H)$ и $\sigma(E, H)$ в условиях фотовозбуждения и $T=4.2 \mathrm{~K}$ представлены на рис. 1,2 . Из рисунков видно, что в квантовых магнитных полях зависимости $R_{\mathrm{H}}(E)$ имеют следующие особенности.

1. В области слабых электрических полей с ростом $H$ растет и величина $R_{\mathrm{H}}$ (к сожалению, при $H \gtrsim 10^{4}$ Э эту область практически не удается измерить из-за резкого возрастания сопротивления образцов).

2. В сильных электрических полях наблюдается сначала резкое уменьшение $R_{\mathrm{H}}$ с ростом $E$, эта зависимость тем сильнее, чем больше $H$ (так, для $H \sim 4 \cdot 10^{4}$ Э в образце № $\left.3 R_{\mathrm{H}} \sim E^{-3}\right)$. Однако при дальнейшем росте $E$ эта зависимость ослабляется и становится близкой к $R_{\mathrm{H}} \sim E^{-0.5}$. 
Параметры исследуемых образцов

\begin{tabular}{c|c|c|c|c|c}
\hline $\begin{array}{c}\text { № } \\
\text { образца }\end{array}$ & $\left|N_{d}-N_{a}\right|, \mathrm{cm}^{-3}$ & $N_{d}, \mathrm{~cm}^{-3}$ & $N_{a}, \mathrm{~cm}^{-3}$ & $K, \%$ & $\begin{array}{c}H=0, T=4.2 \mathrm{~K}, \\
\tau_{c}, \mathrm{c}^{-1}\end{array}$ \\
\hline 1 & $5.7 \cdot 10^{12}$ & $7.2 \cdot 10^{12}$ & $1.5 \cdot 10^{12}$ & 21 & $1.3 \cdot 10^{-5}$ \\
2 & $7.2 \cdot 10^{12}$ & $1.2 \cdot 10^{13}$ & $5.4 \cdot 10^{12}$ & 43 & $3.8 \cdot 10^{-6}$ \\
3 & $9.0 \cdot 10^{11}$ & $7.2 \cdot 10^{12}$ & $6.5 \cdot 10^{12}$ & 88 & $2.6 \cdot 10^{-6}$ \\
4 & $5.7 \cdot 10^{12}$ & $1.7 \cdot 10^{13}$ & $1.1 \cdot 10^{13}$ & 62 & $9.2 \cdot 10^{-7}$ \\
5 & $1.3 \cdot 10^{12}$ & $1.0 \cdot 10^{11}$ & $1.4 \cdot 10^{12}$ & 70 & $3.0 \cdot 10^{-6}$ \\
6 & $5.7 \cdot 10^{12}$ & $1.9 \cdot 10^{12}$ & $7.6 \cdot 10^{12}$ & 25 & $4.6 \cdot 10^{-7}$
\end{tabular}

3. Величина электрического поля, начиная с которого выполняется последняя зависимость, растет с увеличением $H$.

Анализируя зависимости $\sigma(E)$ при разных $H$ (рис. 2), следует отметить, что в области квантовых полей $\sigma$ растет с ростом $E$ и тем сильнее, чем больше $H$. Наличие на всех кривых участка насыщения $\sigma(E)$ связано с тем,

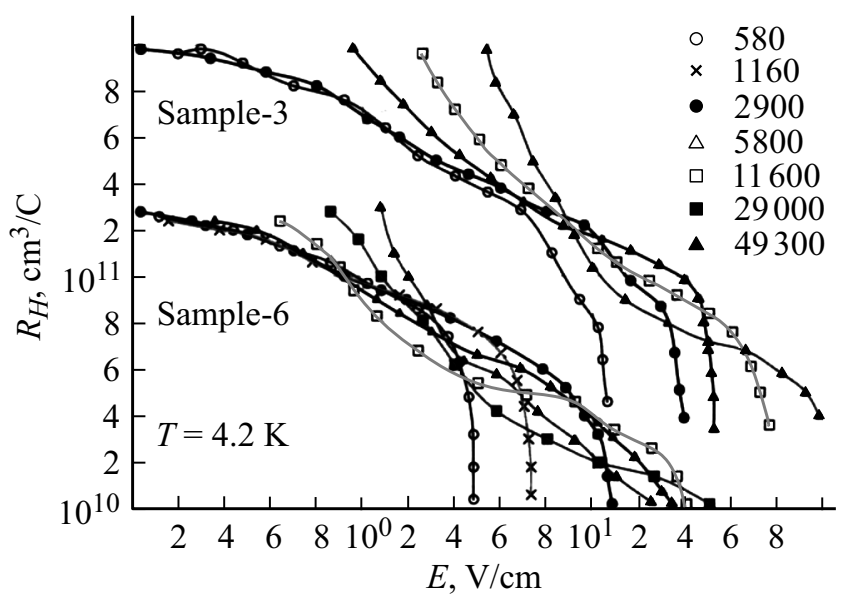

Рис. 1. Зависимости $R_{H}(E)$ для образцов $n$-Ge (№ 3 ) и $p$-Ge (№ 6) в сильных электрических и квантовых магнитных полях.

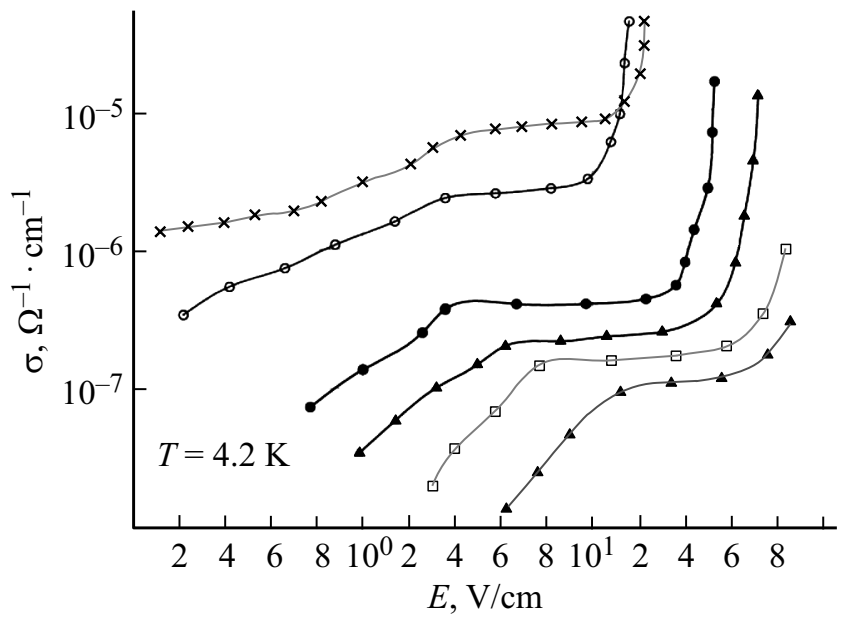

Рис. 2. Зависимости $\sigma(E)$ для образца $n$-Ge (№ 3) для разных $H$. (Обозначения экспериментальных точек, соответствующих разным $H$, такие же, как и на рис. 1$)$.

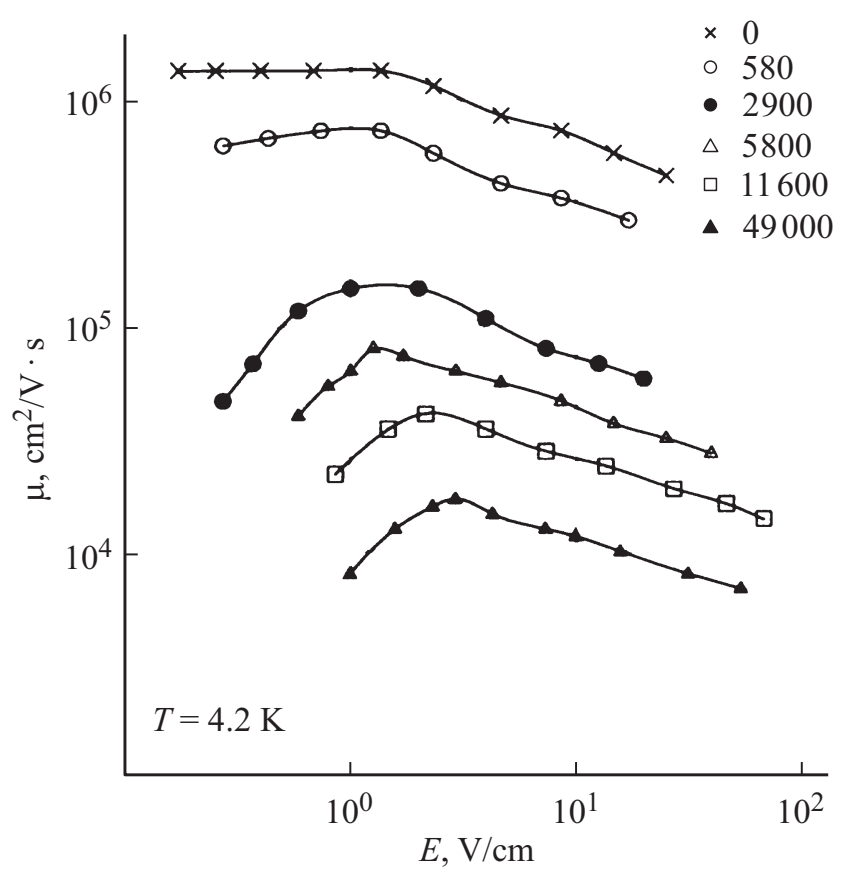

Рис. 3. Зависимости $\mu(E)$ для образца $n$-Ge (№ 3) при разных значениях $H . H$,Э: $1-0,2-580,3-2900,4-5800$, $5-11600,6-49000$.

что в этой области подвижность $\mu \sim E^{-0.5}$ (см. рис. 3), что соответствует рассеянию импульса носителей на акустических фононах.

При фотовозбуждении, как следует из уравнения (2), зная $\left|N_{d}-N_{a}\right|$, можно найти зависимость $\left\langle\tau_{c}\right\rangle_{H}(E)$, которая будет обратной $R_{H}(E)$. Характерные кривые $\left\langle\tau_{c}\right\rangle_{H}(E)$ для образцов $n$ - и $p$-Ge представлены на рис. 4. С ростом $E\left\langle\tau_{c}\right\rangle_{H}$ растет, причем тем значительнее, чем больше $H$, абсолютные значения $\left\langle\tau_{c}\right\rangle_{H}$ уменьшаются, причем тем значительнее, чем меньше $E$.

Эти особенности поведения $\left\langle\tau_{c}\right\rangle_{H}$ хорошо видны на кривых $\left\langle\tau_{c}\right\rangle_{H}(H)$ при разных значениях $E$, представленных для нескольких образцов на рис. 5, $a-c$. Видно, что общий характер кривых для всех образцов одинаков: при $H<H_{\mathrm{qu}}\left\langle\tau_{c}\right\rangle_{H}$ практически не зависит от магнитного поля, при $H>H_{\text {qu }}\left\langle\tau_{c}\right\rangle_{H}$ падает с ростом $H$, абсолютное значение $\left\langle\tau_{c}\right\rangle_{H}$ (при одном и том же $H$ ) растет с увеличением $E$, при $E \gtrsim 10 \mathrm{~B} / \mathrm{cm}\left\langle\tau_{c}\right\rangle_{H}$ практически не зависит от $H$ во всем диапазоне измеряемых значений. 


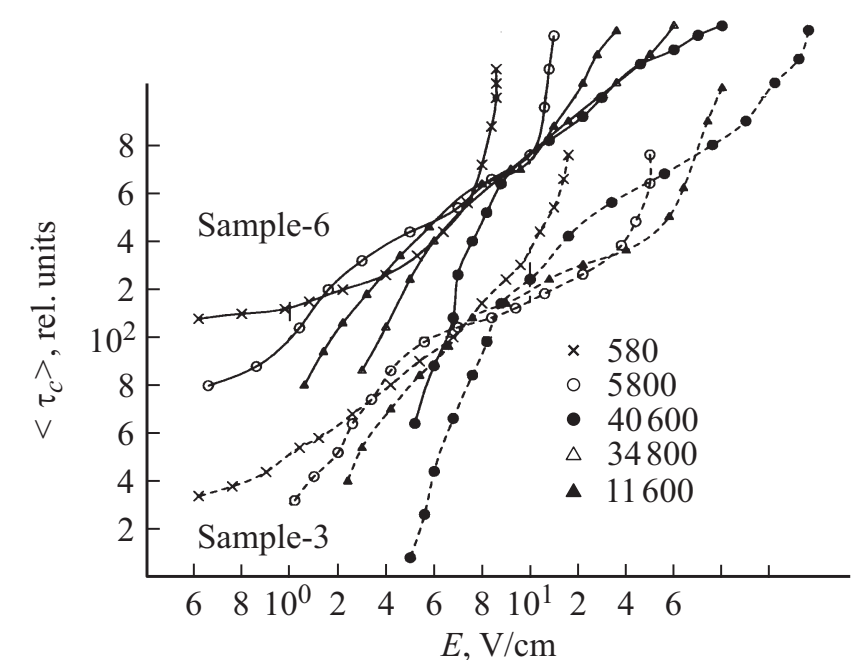

Рис. 4. Зависимости $\langle\tau\rangle_{c}(E)$ для образцов (№ 3$)$ и $p$-Ge (№ 6) при разных значениях $H$.

\section{3. Обсуждение представленных экспериментальных результатов}

1. Обсуждение представленных экспериментальных результатов сосредоточим в основном на поведении $\left\langle\tau_{c}\right\rangle_{H}(E, H)$, так как в условиях фонового подсвета зависимости $R_{\mathrm{H}}(E, H)$ полностью обусловлены поведением $\left\langle\tau_{c}\right\rangle_{H}$. Ход кривых $\sigma(E, H)$ будет в дальнейшем также прокоментирован.
Согласно теории каскадного захвата носителей заряда на изолированный притягивающий центр [6-8] происходит испускание характерного фонона, величина которого в квантующих магнитных полях порядка $\sqrt{\hbar \omega m s^{2}}$, что больше соответствующего фонона в отсутствие $H-\sqrt{k_{0} T m s^{2}},\left(\hbar \omega \gg k_{0} T\right)$. При этом процесс захвата носителя заряда в магнитном поле существенно зависит от соотношения тепловой энергии $k_{0} T$ и энергии характерного фонона. Наиболее интересными являются следующие случаи:

1) $k_{0} T \gg\left(\sqrt{\hbar \omega m s^{2}}\right.$

2) $m s^{2} \ll k_{0} T \ll \sqrt{\hbar \omega m s^{2}}$,

3) $k_{0} T \ll \sqrt{\hbar \omega m s^{2}}, k_{0} T \ll m s^{2}$.

В первом случае электрон с ростом $H$ испускает фононы все большей энергии, темп его энергетической релаксации увеличивается, соответственно время жизни носителей падает.

Отношение $\left\langle\tau_{c}\right\rangle_{H}$ и $\left\langle\tau_{c}\right\rangle-$ равновесное время жизни - в этом случае равно

$$
\frac{\left\langle\tau_{c}\right\rangle_{H}}{\left\langle\tau_{c}\right\rangle}=\left(\frac{8 k_{0} T}{\hbar \omega}\right)\left(\ln \frac{4 k_{0} T}{\sqrt{3 m s^{2} \hbar \omega}}\right)^{-1} .
$$

Во втором случае магнитное поле так велико, что взаимодействие носителей с решеткой неупругое, носитель теперь может испустить крупный акустический фонон. Но так как такой процесс может реализоваться в достаточной близости от центра, то более вероятным оказывается механизм рекомбинации с испусканием теп-

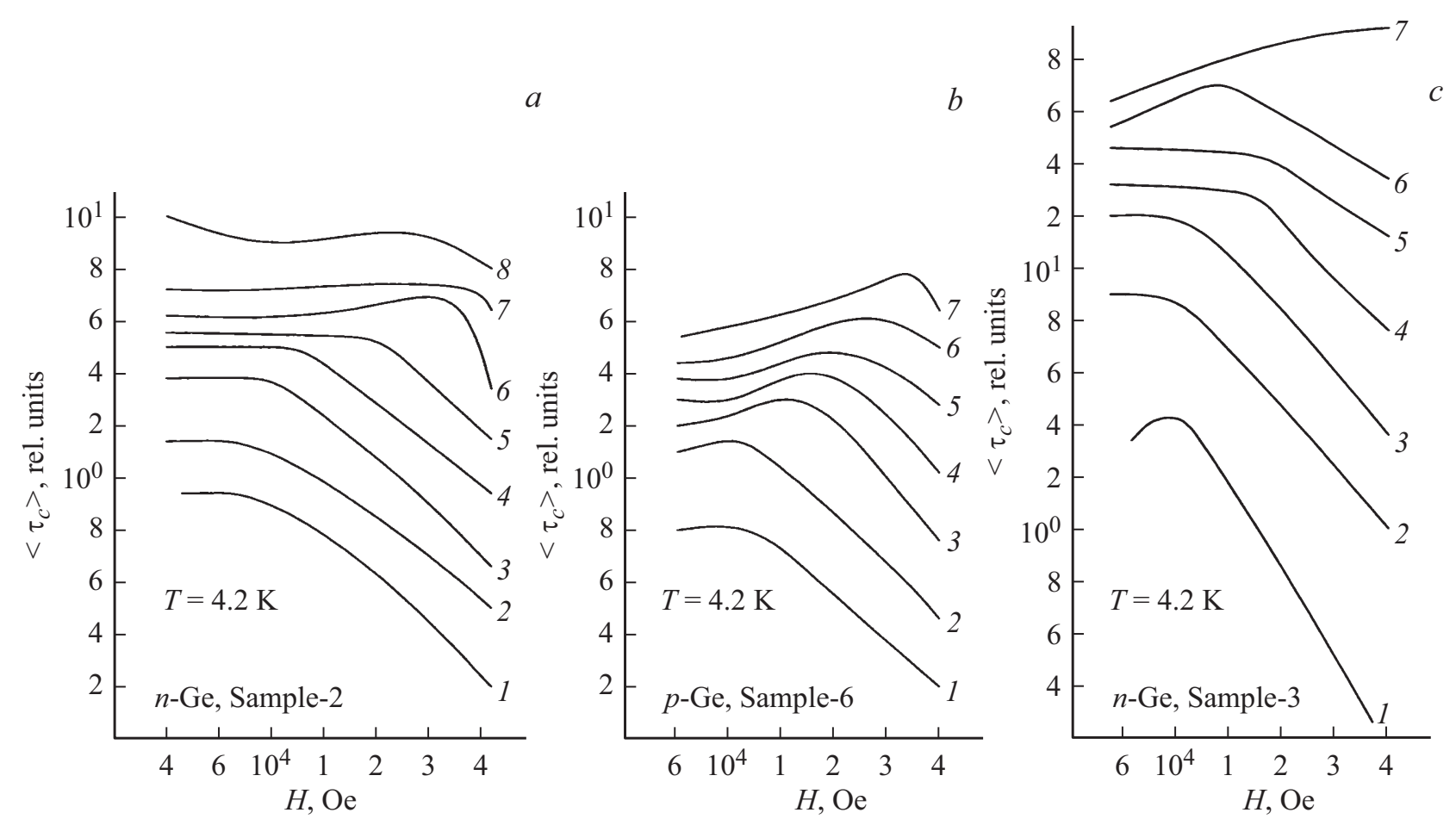

Рис. 5. Зависимости $\langle\tau\rangle_{c}(H)$ для трех образцов германия при разных значениях $E$, В/см. $\left.a\right) 1-0.5,2-1,3-3,4-5,5-6$, $6-8,7-10,8-18$. b) $1-0.5,2-1,3-1.4,4-2,5-3,6-5,7-10$. c) $1-0.5,2-1,3-1.4,4-2,5-3$, $6-5,7-7$. 
ловых фононов. Поэтому в этих условиях время жизни перестает зависеть от $H$, хотя по абсолютной величине остается много меньше равновесного значения.

В этом случае теория дает следующее соотношение времен

$$
\frac{\left\langle\tau_{c}\right\rangle_{H}}{\left\langle\tau_{c}\right\rangle}=\left(\frac{m s^{2}}{k_{0} T}\right)^{2} \frac{16}{3} .
$$

Третий случай - очень низких температур и квантовых магнитных полей, при которых захват носителя возможен только в поле центра. Расчеты показывают, что здесь, как и во втором случае, $\left\langle\tau_{c}\right\rangle_{H}$ не зависит от $H$, но величина его больше равновесного значения

$$
\frac{\left\langle\tau_{c}\right\rangle_{H}}{\left\langle\tau_{c}\right\rangle}=0.7 \frac{m s^{2}}{k_{0} T} .
$$

2. Чтобы провести сопоставление экспериментальных данных с расчетом, выполним некоторые оценки с учетом конкретных условий, в которых выполнялся эксперимент.

\section{n-Ge}

$T=4.2 \mathrm{~K}, \quad m=0.12 m_{0}, \quad k_{0} T=3.6 \cdot 10^{-4}$ эВ, $\sqrt{k_{0} T m s^{2}}=0.85 \cdot 10^{-4}$ эВ, магнитное поле, при котором $\hbar \omega=k_{0} T$ равно $H=3.7 \cdot 10^{3} \ni$. Будем считать, что квантовым пределом является магнитное поле, для которого $\hbar \omega \geq 5 k_{0} T$, что соответствует $H_{\mathrm{qu}} \geq 20$ кЭ.

С другой стороны, величина фонона в магнитном поле $H=20$ кЭ составляет $\sqrt{\hbar \omega m s^{2}} \simeq 2 \cdot 10^{-4}$ эВ, т.е. сравнима с величиной $k_{0} T$ во всем интервале реализуемых в эксперименте магнитных полей $(H=4-50$ кЭ, $\sqrt{\hbar \omega m s^{2}}=(0.9-3.1) \cdot 10^{-4}$ эВ).

\section{p-Ge}

$m=0.22 m_{0}, \sqrt{k_{0} T m s^{2}}=1.15 \cdot 10^{-4}{ }_{\ni} \mathrm{B}, \hbar \omega=k_{0} T$ при $H=6.8 \cdot 10^{3}$ Э квантовый предел $\left(\hbar \omega \geq 5 k_{0} T\right)$ при $H_{\mathrm{qu}} \geq 35$ кЭ; $\sqrt{\hbar \omega m s^{2}}=2.6 \cdot 10^{-4}{ }_{\ni} \mathrm{B}$.

Таким образом, в $p$-Ge так же, как и в $n$-типе, во всем диапазоне реализуемых в эксперименте $H$, $\sqrt{\hbar \omega m s^{2}} \approx k_{0} T$, т.е. в условиях нашего эксперимента результаты должны быть ближе всего к случаю „а“.

Обсудим с этой точки зрения полученные экспериментальные результаты.

3. Учитывая теоретические результаты [6-9], в условиях нашего эксперимента следует ожидать.

- В слабых электрических полях, но в квантующих магнитных полях $\left\langle\tau_{c}\right\rangle_{H} \sim H^{-2}$ и $\left\langle\tau_{c}\right\rangle_{H} \sim T^{2}$.

- Электрические поля, используемые в эксперименте, как показывают оценки, не снимают квантования. Поэтому греющее электрическое поле должно приводить к росту $\left\langle\tau_{c}\right\rangle_{H} \sim E^{3}$ (при фиксированном $H$ ) и уменьшению $\left\langle\tau_{c}\right\rangle_{H}$ с ростом $H$ по закону $\left\langle\tau_{c}\right\rangle_{H} \sim H^{-6}$.

Из рис. $5, a-c$ и 6 видно, что действительно при

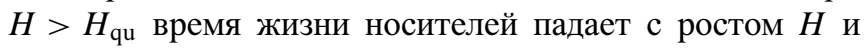
растет с повышением температуры. Но если температурный закон изменения $\left\langle\tau_{c}\right\rangle_{H}$ близок к расчетному, то зависимость $\left\langle\tau_{c}\right\rangle_{H}$ от магнитного поля слабее квадратичной.

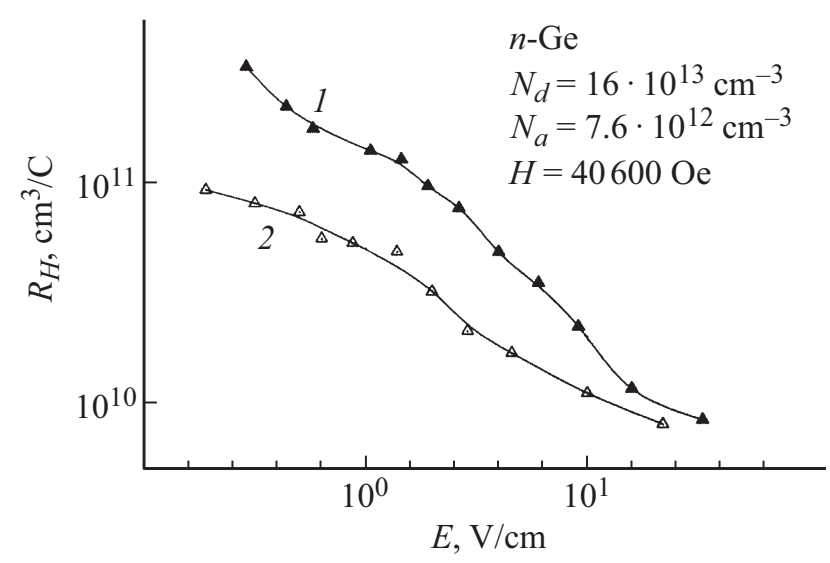

Рис. 6. Зависимость $R_{\mathrm{H}}(E)$ для $n-\mathrm{Ge}$ (образец № 4$)$ в квантовом магнитном поле $(H=40600 Э)$ при разных значениях $T$. $T, \mathrm{~K}: 1-1.8,2-4.2$.

Эти отклонения, по-видимому, связаны с тем, что в эксперименте не выполняется строгое неравенство $k_{0} T \gg \sqrt{\hbar \omega m s^{2}}$. При его невыполнении $\left\langle\tau_{c}\right\rangle_{H}$ стремится к насыщению с ростом $H$; это может привести к ослаблению зависимости $\left\langle\tau_{c}\right\rangle_{H}(H)$ по сравнению с расчетной.

Что касается $p$-Ge, то в нем хуже, чем в $n$-типе, в условиях нашего эксперимента выполняются требования теории, поэтому можно наблюдать только качественное согласие.

Электрические поля, используемые в эксперименте, как показывают оценки, не снимают квантование. Поэтому греющее электрическое поле должно приводить к росту $\left\langle\tau_{c}\right\rangle_{H}$ по закону $\left\langle\tau_{c}\right\rangle_{H} \sim E^{3}$ (при фиксированном $H$ ) и к уменьшению $\left\langle\tau_{c}\right\rangle_{H}$ с ростом $H$ по закону $\left\langle\tau_{c}\right\rangle_{H} \sim H^{-6}$.

Из рис. 4 видно, что в $H \sim 40$ КЭ (что соответствует $\left.\frac{\hbar \omega_{c}}{k_{0} T} \simeq 10\right)$ зависимость $\left\langle\tau_{c}\right\rangle_{H}(E)$ действительно близка к кубической.

С другой стороны, из рис. 5 видно, что увеличение электрического поля не приводит к заметному усилению $\left\langle\tau_{c}\right\rangle_{H}(H)$, в лучшем случае она становится близка к квадратичной. Это, возможно, связано с тем, что наличие электрического поля приводит к частичному снятию квантования, и весь диапазон реализуемых в эксперименте магнитных полей оказывается меньше $H_{\mathrm{qu}}$ квантового предела. Кроме того, в теории использовалось предположение только акустического рассеяния энергии и импульса носителей.

Однако в используемых в эксперименте электрических полях возможен вклад в рассеяние взаимодействия с оптическими фононами $[3,4]$ (особенно в $p$-Ge), что также может привести к ослаблению зависимости $\left\langle\tau_{c}\right\rangle_{H}(E)$. Ход кривых $\sigma(E)$ в разных $H$ (рис. 2) становится понятным из сопоставления зависимостей $R_{\mathrm{H}}(E)$ (рис. 1) и $\mu(E)$ (рис. 3). Увеличение $\sigma$ с ростом $E$ связано с зависимостью $\left\langle\tau_{c}\right\rangle_{H}(E)$, уменьшение $\sigma$ с ростом $H$ связано с наличием магнитосопротивления (см. рис. $3 \mu(E)$ в разных $H)$. Участок насыщения $\sigma(E)$, так называемый „квазион“, объясняется тем, что в сильных 
электрических полях $\left\langle\tau_{c}\right\rangle_{H}(E)$ и $\mu_{H}(E)$ имеют противоположные законы изменения:

$$
\left\langle\tau_{c}\right\rangle_{H}\left(H \sim E^{0.5}, \mu_{H} \sim E^{-0.5}\right.
$$

\section{4. Заключение}

Таким образом, в работе подробно рассмотрены особенности разогрева носителей заряда в чистом германии $n$ - и $p$-типов электрическим полем в квантующих магнитных полях в условиях фотогенерации носителей. Получены следующие результаты.

- При фотовозбуждении носителей, при котором зависимости $n$ и $p$ от $E$ и $H$ соответствуют зависимостям времени жизни от соответствующих полей, в слабых электрических полях $\left\langle\tau_{c}\right\rangle_{H}$ практически не зависит от магнитного поля и уменьшается с ростом $H$ при $H \geq H_{\mathrm{qu}}$.

- По мере роста электрического поля абсолютные значения $\left\langle\tau_{c}\right\rangle_{H}$ (при фиксированном $H \geq H_{\mathrm{qu}}$ растут, а также увеличивается область магнитных полей, для которых $\left\langle\tau_{c}\right\rangle_{H}$ не зависит от $H$.

- Понижение температуры усиливает названные процессы.

- Наблюдаемые в эксперименте зависимости $\left\langle\tau_{c}\right\rangle_{H}(E, H)$, можно аппроксимировать степенными функциями со следующими значениями показателей степени: $\left\langle\tau_{c}\right\rangle_{H} \sim H^{-1}$ и $\left\langle\tau_{c}\right\rangle_{H} \sim E^{2}$.

- Полученные результаты качественно согласуются с теорией захвата носителей заряда в скрещенных электрическом и магнитном полях, разработанной в [6-9].

\section{Список литературы}

[1] В.Ф. Банная, Л.И. Веселова, Е.М. Гершензон, В.А. Чуенков. ФТП, 10 (2), 338 (1976).

[2] В.Ф. Банная, Л.И. Веселова, Е.М. Гершензон, В.А. Чуенков. ФТП, 7 (10), 1972 (1973).

[3] Л.И. Веселова. Автореф. канд. дис. (М., 1978).

[4] В.Ф. Банная, Т.Г. Фукс, Ю.П. Ладыжинский. ФТП, 7 (10), 1978 (1973).

[5] В.Ф. Банная, Т.Г. Фукс, Ю.П. Ладыжинский. ФТП, 7 (8), 1507 (1973).

[6] В.Н. Абакумов, Л.Н. Крещук, И.Н. Яссиевич. ФТП, 12 (2), 264 (1978).

[7] В.Н. Абакумов, Л.Н. Крещук, И.Н. Яссиевич. ЖЭТФ, 74, 1020 (1978).

[8] В.Н. Абакумов, Л.Н. Крещук, И.Н. Яссиевич. ЖЭТФ, 75, 1342 (1978).

[9] Л.Н. Крещук. ФТП, 13, 919 (1979).

Редактор Г.А. Оганесян

\section{Influence of a quantum magnetic field on the heating of charge carriers in pure Ge}

\author{
V.F. Bannaya ${ }^{1}$, E.V. Nikitina ${ }^{2}$ \\ ${ }^{1}$ Moscow State University of Education, \\ 119991 Moscow, Russia \\ 2 Peoples'Friendship Unieversity of Russia \\ (RUDN University), \\ 117198 Moscow, Russia
}

Abstract The results of an experimental study of the heating of charge carriers by an electric field $\mathbf{E}$ in pure $n$ - and p-types germanium in a quantizing magnetic field $(\mathbf{H})$ at $\mathbf{E} \perp \mathbf{H}$ under the conditions of photoexcitation of the carriers are under detailed consideration. We was obtained results are in qualitative agreement with the theory of charge carriers capture in crossed electric and magnetic fields. 